STRUCTURAL BIOLOGY

ISSN 2059-7983

Received 29 November 2016

Accepted 14 March 2017

Edited by R. McKenna, University of

Florida, USA

₹ These authors contributed equally to this work.

Keywords: X-ray diffraction; molecular replacement; acyl binding; Oryza sativa; isothermal titration calorimetry.

PDB references: OsACBP1, 5h3g; OsACBP2, $5 \mathrm{~h} 3 \mathrm{i}$

Supporting information: this article has supporting information at journals.iucr.org/d

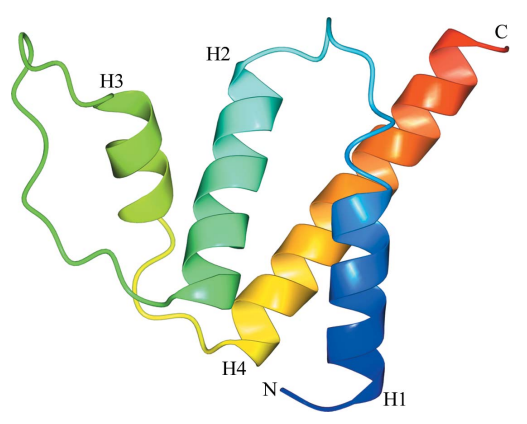

C 2017 International Union of Crystallography

\section{The first plant acyl-CoA-binding protein structures: the close homologues OsACBP1 and OsACBP2 from rice}

\author{
Ze-Hua Guo, ${ }^{a} \neq$ Wallace H. Y. Chan, ${ }^{a} \neq$ Geoffrey K. W. Kong, ${ }^{a *}$ Quan Hao ${ }^{b}$ and \\ Mee-Len Chye ${ }^{\text {a* }}$
}

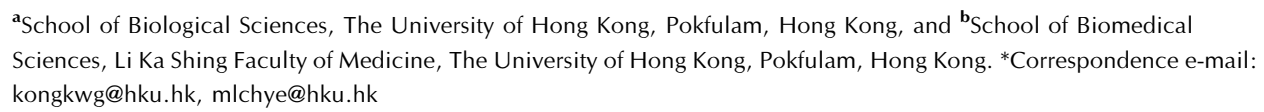

Acyl-CoA-binding proteins (ACBPs) are a family of proteins that facilitate the binding of long-chain acyl-CoA esters at a conserved acyl-CoA-binding domain. ACBPs act to form intracellular acyl-CoA pools, transport acyl-CoA esters and regulate lipid metabolism. In the model plant Arabidopsis thaliana, a family of six ACBPs has been demonstrated to function in stress and development. Six ACBPs (OsACBPs) have also been identified in Oryza sativa (rice), but they are not as well characterized as those in Arabidopsis thaliana. To understand the need in rice for the two $10 \mathrm{kDa}$ ACBPs, namely OsACBP1 and OsACBP2, which share $79 \%$ sequence identity, their crystal structures were elucidated and their affinities toward acyl-CoA esters were compared using isothermal titration calorimetry. OsACBP2 was found to display a higher binding affinity for unsaturated acyl-CoA esters than OsACBP1. A difference between the two proteins is observed at helix 3 and is predicted to lead to different ligand-binding modes in terms of the shape of the binding pocket and the residues that are involved. OsACBP1 thus resembles bovine ACBP, while OsACBP2 is similar to human liver ACBP, in both structure and binding affinity. This is the first time that ACBP structures have been reported from plants, and suggests that OsACBP1 and OsACBP2 are not redundant in function despite their high sequence identity and general structural similarity.

\section{Introduction}

Fatty acids must be esterified with coenzyme A by acyl-CoA synthase before they can be used as fuel or modified to synthesize other lipid products (Rasmussen et al., 1994). AcylCoA-binding proteins (ACBPs), which bind acyl-CoA esters, can thus maintain an intracellular acyl-CoA pool and transport acyl-CoA esters in lipid metabolism. These essential proteins have been found in all eukaryotes examined, as well as in 11 eubacterial species (Burton et al., 2005), in addition to being widely distributed in the plant kingdom (Meng et al., 2011).

Plant ACBPs were divided into four classes (I-IV) after the discovery of various ACBP homologues with common functional domains in Arabidopsis thaliana (AtACBPs) and Oryza sativa (OsACBPs) (Leung et al., 2004; Meng et al., 2011). Phylogenetic, gene expression and biochemical analyses suggest that paralogues within and across the four classes are not redundant proteins (Meng et al., 2011). In rice, OsACBP1 (locus ID Os08g0162800) and OsACBP2 (locus ID Os06g0115300) belong to class I (Meng et al., 2011), and this class, which is present in virtually all plant species for which 
genomes have been sequenced, contains only the acyl-CoAbinding (ACB) domain and is most closely related to the $\sim 10 \mathrm{kDa}$ prototype that has been well characterized in the human liver and bovine ACBPs, amongst others (van Aalten et al., 2001; Taskinen et al., 2007). OsACBP3 (locus ID Os03g0576699) is also grouped in class I, although it contains an extension of 64 residues after the ACB domain. Other classes (II-IV) of ACBPs in plants are larger as they contain additional domains, including transmembrane domains, Kelch motifs or ankyrin repeats (Meng et al., 2011). In fact, these domains represent a major criterion used in the classification of ACBPs within the plant kingdom (Meng et al., 2011).

The biochemistry of plant ACBPs has been studied using recombinant proteins in in vitro binding assays (Engeseth $e t$ al., 1996; Chye, 1998, 2000; Leung et al., 2004, 2006; Chen et al., 2008, 2010; Gao et al., 2009; Xiao et al., 2009; Du et al., 2010; Meng et al., 2011, 2014; Hsiao et al., 2014; Xue et al., 2014). Recombinant Brassica napus $10 \mathrm{kDa}$ ACBP was found to bind oleoyl-CoA (18:1-CoA) and palmitoyl-CoA (16:0-CoA) very strongly, similar to mammalian and yeast ACBPs, in a 1:1 stoichiometric ratio (Engeseth et al., 1996). Heterologous expression of this Brassica ACBP altered acyl-CoA pool composition in Arabidopsis (Yurchenko et al., 2009, 2014). All six AtACBPs have been found to bind 16:0-CoA, 18:1-CoA, linoleoyl-CoA (18:2-CoA) and linolenoyl-CoA (18:3-CoA) as well as phosphatidylcholine (PC). Some AtACBPs have individual specificities for other acyl-CoAs and lipids: $\mathrm{His}_{6}$ AtACBP1 displayed affinity for very long chain acyl-CoA esters (24:0-CoA, 25:0-CoA and 26:0-CoA) as well as for phosphatidic acid (Du et al., 2010; Xue et al., 2014), while $\mathrm{His}_{6^{-}}$ AtACBP2 could bind to lysoPC (Gao et al., 2010) and $\mathrm{His}_{6}$-AtACBP3 to arachidonyl-CoA as well as phosphatidylethanolamine (PE; Xiao et al., 2010). Point mutations in the acyl-CoA-binding domain of AtACBPs led to decreased affinity for acyl-CoA esters, thus confirming the lipid-binding function of the acyl-CoA-binding domain (Chye et al., 2000; Leung et al., 2004, 2006).

Arabidopsis ACBPs show nonredundant biological functions in vivo. AtACBP4, AtACBP5 and AtACBP6 have been found to be localized in the cytosol (Chen et al., 2008; Xiao, Li et al., 2008) and can maintain a cytosolic acyl-CoA pool. Single-gene knockout mutants of AtACBP4, AtACBP5 and AtACBP6 displayed no significant changes in visible phenotype, although the AtACBP4 mutant showed an abnormal lipid composition in the leaf membrane (Xiao, Li et al., 2008), while seed weight was reduced in the AtACBP4-AtACBP5AtACBP6 mutant (Hsiao et al., 2014). These three cytosolic AtACBPs also cooperate in acyl-lipid metabolism during pollen development (Hsiao et al., 2014). Overexpression of AtACBP6 conferred freezing tolerance in rosette leaves and flowers (Chen et al., 2008; Liao et al., 2014). AtACBP1 and AtACBP2 have been localized in the endoplasmic reticulum and plasma membrane (Chye et al., 1999; Li \& Chye, 2003), while AtACBP3 is apoplast-targeted and membrane-associated (Leung et al., 2006). AtACBP1 plays roles in lead tolerance (Xiao, Gao et al., 2008), embryogenesis (Chen et al., 2010; Du et al., 2013b), seed germination and seedling development (Du et al., 2013b), and cuticle biosynthesis (Xue et al., 2014). AtACBP2 has been shown to mediate cadmium and oxidative stress and confer drought tolerance (Gao et al., 2009; Du et al., 2013a), whereas AtACBP3 has been found to have a function in autophagy-mediated leaf senescence and pathogen resistance (Xiao et al., 2010; Xiao \& Chye, 2011).

Recombinant Oryza sativa ACBPs have also been reported to bind acyl-CoA esters in Lipidex assays (Meng et al., 2011). $\mathrm{His}_{6}$-OsACBP1 was reported to bind 18:1-CoA, 18:2-CoA, 18:3-CoA and 16:0-CoA (Meng et al., 2011). The two other class I ACBPs, $\mathrm{His}_{6}-\mathrm{OsACBP} 2$ and $\mathrm{His}_{6}$-OsACBP3, were reported to bind 18:3-CoA. $\mathrm{His}_{6}-\mathrm{OsACBP} 5$ and $\mathrm{His}_{6^{-}}$ OsACBP6 bound 16:0-CoA and 18:1-CoA, respectively, in addition to 18:3-CoA, while $\mathrm{His}_{6}-\mathrm{OsACBP}_{4}$ was shown to bind 16:0-CoA, 18:2-CoA and 18:3-CoA (Meng et al., 2011). The differential binding affinities for acyl-CoA esters again suggest that the rice ACBPs probably assume different roles in vivo.

The ACB domain is the sole domain in OsACBP1 and OsACBP2 (Meng et al., 2011). OsACBP1 mRNA is expressed at similar levels during the anthesis, milk and soft-dough stages of development, while $O S A C B P 2$ mRNA peaks at the dough stage (Meng et al., 2011). The subcellular localizations of the six OsACBPs were determined using OsACBP::green fluorescent protein (GFP) fusion proteins by confocal microscopy (Meng et al., 2014). OsACBP1::GFP and OsACBP2::GFP were localized to the cytosol, but OsACBP3::GFP seemed to be localized to multiple compartments (Meng et al., 2014). OsACBP1 and OsACBP2 are thus more conserved among the three class I OsACBPs with respect to localization and domain structure (Meng et al., 2014).

The ACB domain common to all ACBPs is the most structurally conserved part of the ACBPs. The first ACBP structure was elucidated from the $9.9 \mathrm{kDa}$ bovine ACBP using nuclear magnetic resonance spectroscopy (Andersen \& Poulsen, 1993). The ACB domain structure consists of four $\alpha$-helices in an up-down-down-up arrangement, in which helices 1, 2 and 4 (numbered from the N-terminus) are more closely packed together and helix 3 is in close contact with helix 2 only (Andersen \& Poulsen, 1993). This helical scaffold, which is unique to ACBPs, was conserved in all ACBP structures determined subsequently, namely those from yeast (Teilum et al., 2005), human liver (Taskinen et al., 2007), armadillo (Costabel et al., 2006), Plasmodium falciparum (van Aalten et al., 2001) and the plant fungus Moniliophthora perniciosa (Monzani et al., 2010). Interestingly, the ACBP from $M$. perniciosa contains an additional fifth helix at the C-terminus; the first, second, fourth and fifth helices form a classical four-helix bundle, while the first, second, third and fourth helices belong to the classical ACBP scaffold (Monzani et al., 2010). The ligand-binding modes among ACBPs are diverse; for example, human liver and M. perniciosa ACBPs undergo dimerization (Taskinen et al., 2007; Monzani et al., 2010), while bovine ACBP remains monomeric but shows a slight overall structural tightening and binds acyl-CoA esters with the acyl moiety extending in an opposite direction to that observed in human liver ACBP (Kragelund et al., 1993). 
Table 1

X-ray diffraction data-collection and crystallographic refinement statistics.

Values in parentheses are for the highest resolution shell.

\begin{tabular}{|c|c|c|}
\hline & OsACBP1 & OsACBP2 \\
\hline PDB code & $5 \mathrm{~h} 3 \mathrm{~g}$ & $5 \mathrm{~h} 3 \mathrm{i}$ \\
\hline \multicolumn{3}{|l|}{ Data processing } \\
\hline Space group & $P 322_{1}$ & $P 2_{1} 2_{1} 2$ \\
\hline Unit-cell parameters $(\AA)$ & $\begin{array}{c}a=b=59.68 \\
c=60.72\end{array}$ & $\begin{array}{c}a=100.57, b=145.05 \\
\quad c=25.89\end{array}$ \\
\hline No. of crystals & 1 & 1 \\
\hline $\begin{array}{l}\text { No. of molecules per } \\
\text { asymmetric unit }\end{array}$ & 1 & 4 \\
\hline Resolution $(\AA)$ & 25.8-1.60 (1.70-1.60) & $41.3-2.30(2.40-2.30)$ \\
\hline Completeness (\%) & $90.5(49.2)$ & $79.3(31.9)$ \\
\hline$R_{\text {merge }}$ & $0.078(0.835)$ & $0.134(0.312)$ \\
\hline$\langle I / \sigma(I)\rangle$ & $25.9(5.8)$ & $12.0(5.8)$ \\
\hline Multiplicity & $21.3(20.7)$ & $6.78(6.79)$ \\
\hline $\mathrm{CC}_{1 / 2}^{\dagger}$ & $0.999(0.978)$ & $0.997(0.978)$ \\
\hline \multicolumn{3}{|l|}{ Refinement } \\
\hline Wilson $B$ factor $\left(\AA^{2}\right)$ & 15.3 & 20.7 \\
\hline No. of reflections & 15625 & 14099 \\
\hline$R_{\text {work }} / R_{\text {freeł }}$ & $0.191 / 0.234$ & $0.223 / 0.255$ \\
\hline \multicolumn{3}{|l|}{ No. of atoms } \\
\hline Protein & 756 & 2916 \\
\hline Water & 139 & 342 \\
\hline Glycerol & 24 & \\
\hline Chloride ion & 3 & \\
\hline MPD $\$$ & & 32 \\
\hline Acetate & & 4 \\
\hline \multicolumn{3}{|l|}{$\langle B\rangle\left(\AA^{2}\right)$} \\
\hline Protein & 20.5 & 28.3 \\
\hline Water & 38.2 & 36.7 \\
\hline Glycerol & 45.4 & \\
\hline Chloride ion & 24.6 & \\
\hline MPD & & 39.6 \\
\hline Acetate & & 58.4 \\
\hline \multicolumn{3}{|l|}{ R.m.s. deviations } \\
\hline Bond lengths $(\AA)$ & 0.005 & 0.002 \\
\hline Bond angles $\left({ }^{\circ}\right)$ & 0.77 & 0.39 \\
\hline \multicolumn{3}{|l|}{ Ramachandran plot } \\
\hline Favoured (\%) & 100 & 98.3 \\
\hline Acceptable (\%) & 0 & 1.7 \\
\hline Outliers (\%) & 0 & 0 \\
\hline
\end{tabular}

$+\mathrm{CC}_{1 / 2}$ is the Pearson correlation coefficient between independently merged halves of the data set. The highest resolution shell $C_{1 / 2}$ values are significant at $p=0.001$. $\quad 5 \%$ of all reflections were set aside for the free $R$ set. $\$ 2$-Methyl-2,4-pentanediol.

Although the structures of ACBPs from various eukaryotes have been solved, these do not include any from plants. In this paper, the structures of two $10 \mathrm{kDa}$ rice ACBPs, OsACBP1 and OsACBP2, which share $79 \%$ amino-acid identity, are presented.

\section{Materials and methods}

\subsection{Expression and purification of OsACBP1 and OsACBP2}

The procedure of Meng et al. (2011) was optimized for the expression of recombinant OsACBP1 and OsACBP2 with an $\mathrm{N}$-terminal $\mathrm{His}_{6}$ tag from Escherichia coli BL21 (DE3) Star pLysS (Invitrogen) cells transformed with plasmids pOS502 and pOS503, respectively, which were derived from the pRSETA vector (Life Technologies). The cells were grown at $310 \mathrm{~K}$ in 1.5-3 1 Luria Broth (Sigma) supplemented with $100 \mu \mathrm{g} \mathrm{ml}^{-1}$ ampicillin and $34 \mu \mathrm{g} \mathrm{ml}^{-1}$ chloramphenicol and induced with $0.1 \mathrm{~m} M$ isopropyl $\beta$-D-1-thiogalactopyranoside
(IPTG) for 3-4 $\mathrm{h}$ after the optical density at $600 \mathrm{~nm}\left(\mathrm{OD}_{600}\right)$ had reached 0.6. The cells were then harvested by centrifugation at $4000 \mathrm{~g}$ for $15 \mathrm{~min}$ at $277 \mathrm{~K}$ and the pellets were resuspended in $20 \mathrm{mM} \mathrm{NaH} \mathrm{PO}_{4}, 300 \mathrm{~m} M \mathrm{NaCl}, 50 \mathrm{~m} M$ imidazole (final $\mathrm{pH}$ values of 8.0 for $\mathrm{His}_{6}-\mathrm{OsACBP} 1$ and 7.5 for $\mathrm{His}_{6}$-OsACBP2) prior to sonication. The supernatant was extracted by centrifugation at $40000 \mathrm{~g}$ for $30 \mathrm{~min}$, further clarified by centrifugation at $40000 \mathrm{~g}$ for $20 \mathrm{~min}$ and then passed through $0.22 \mu \mathrm{m}$ PES membrane syringe filters (Millipore) before being applied onto a HisTrap HP column (GE Healthcare) charged with $0.1 M \mathrm{NiCl}_{2}$. The column was washed with $20 \mathrm{mM} \mathrm{NaH} \mathrm{PO}_{4}, 300 \mathrm{mM} \mathrm{NaCl}, 50 \mathrm{~m} M$ imidazole to remove nonspecifically bound proteins from the column before $20 \mathrm{mM} \mathrm{NaH} \mathrm{PO}_{4}, 300 \mathrm{~m} M \mathrm{NaCl}, 300 \mathrm{~m} M$ imidazole was applied to elute the proteins. His ${ }_{6}$-OsACBP1 was further purified by anion-exchange chromatography using an $\mathrm{NaCl}$ gradient from $5 \mathrm{~m} M$ to $1.0 M$ buffered with $20 \mathrm{~m} M$ Tris $\mathrm{pH} 8.0$ on a HiTrap Q column (GE Healthcare). His $6^{-}$ OsACBP2 was further purified by gel-filtration chromatography on a HiLoad 16/600 Superdex 75 pg column (GE Healthcare) equilibrated with $20 \mathrm{~m} M$ Tris, $100 \mathrm{~m} M \mathrm{NaCl}$ $\mathrm{pH} 7.5$.

\subsection{Crystallization and data collection}

Both $\mathrm{His}_{6}-\mathrm{OsACBP} 1$ and $\mathrm{His}_{6}-\mathrm{OsACBP} 2$ were exchanged into $20 \mathrm{~m} M$ Tris, $5 \mathrm{~m} M \mathrm{NaCl}$ (final $\mathrm{pH}$ 8.0) and concentrated for crystallization using Amicon Ultra centrifugal filter devices (Millipore). Initial crystallization conditions were identified through screening in 96-well plates using Crystal Screen and Crystal Screen 2 (Hampton Research), Index Screen (Hampton Research) and The PEGs II Suite (Qiagen), and were further optimized using the vapour-diffusion method in 24-well plates at room temperature. The best diffracting crystals of $\mathrm{His}_{6}$-OsACBP1 were obtained in hanging drops formed by mixing $1 \mu \mathrm{l}$ protein solution at 10 or $20 \mathrm{mg} \mathrm{ml}^{-1}$ with $1 \mu \mathrm{l}$ reservoir solution consisting of $0.10 M$ citric acid $\mathrm{pH}$ 3.8, 2.5-2.6 $M \mathrm{NaCl}$. For $\mathrm{His}_{6}$-OsACBP2 the best crystals were obtained in hanging drops formed by mixing $0.4 \mu \mathrm{l}$ protein solution at $30 \mathrm{mg} \mathrm{ml}^{-1}$ with $1 \mu \mathrm{l}$ reservoir solution consisting of $0.10 \mathrm{M}$ sodium acetate $\mathrm{pH} 4.5-4.7,26-27 \%(w / v)$ polyethylene glycol with an average molecular mass of 4000 (PEG 4000), $0.10 \mathrm{M} \mathrm{MgCl}_{2}$.

Crystals of $\mathrm{His}_{6}-\mathrm{OsACBP1}$ were prepared for diffraction data collection by transferring them successively through drops of reservoir solution supplemented with 10, 20 and finally $30 \%(v / v)$ glycerol for several minutes each before direct plunging them in a loop into liquid nitrogen. The $\mathrm{His}_{6}-\mathrm{OsACBP} 2$ crystals were transferred through drops of reservoir solution supplemented with 10 and $20 \%(v / v)$ 2-methyl-2,4-pentanediol (MPD) before plunging. Diffraction data (temperature $100 \mathrm{~K}, \mathrm{X}$-ray wavelength $0.9900 \AA$ ) were collected on the BL17U beamline at the Shanghai Synchrotron Radiation Facility.

\subsection{Structure solution and analysis}

The diffraction data sets were processed using $X D S$ (Kabsch, 2010), followed by ellipsoidal truncation and aniso- 
tropic scaling using the Diffraction Anisotropy Server (Strong et al., 2006) to correct for the anisotropy present in the data (Table 1). The phases were solved for OsACBP1 by molecular replacement with Phaser (McCoy, 2007) using apo human liver ACBP (Taskinen et al., 2007; PDB entry 2fj9) as the search model. Refinement followed using PHENIX (Adams et al., 2002) with extensive rebuilding of loop regions between helices, aided by the inspection of intermediate models in the molecular-graphics display program Coot (Emsley et al., 2010). The phase solution of OsACBP2 was obtained through molecular replacement using Phaser with OsACBP1 as the search model. The correct molecular-replacement solution could only be obtained with the anisotropy-corrected data, and the space group was confirmed through testing molecular replacement in all related space groups within the primitive orthorhombic lattice. During the refinement of OsACBP2, continual rebuilding of loop regions was required and noncrystallographic symmetry torsion-angle restraints were applied until the final few cycles. In the final stages of refinement solvent molecules were added and translation/libration/ screw (TLS) refinement was incorporated in both structures.

For structural comparisons between the two OsACBPs and with other reported ACBP structures, each of the four molecules of OsACBP2 in the asymmetric unit of the structure and the other structures were superimposed onto OsACBP1 using LSQKAB (Kabsch, 1976) in CCP4 (Winn et al., 2011) and MultiSeq (Roberts et al., 2006) in VMD (Humphrey et al., 1996). Secondary structures were defined using the online version of $D S S P$ (Kabsch \& Sander, 1983). The ligand-binding cavities and residues in OsACBP1 and OsACBP2 were predicted using default parameters by the MetaPocket 2.0 web server (Zhang et al., 2011), which seeks consensus from eight methods.

\subsection{Isothermal titration calorimetry (ITC) experiments}

To investigate the binding of $\mathrm{His}_{6}$-OsACBP1 and $\mathrm{His}_{6}$ OsACBP2 to various acyl-CoA esters (16:0-CoA, 18:2-CoA and 18:3-CoA), titration was carried out with a MicroCal iTC200 system (GE Healthcare) at $303 \mathrm{~K}$. The acyl-CoA esters (Avanti Polar Lipids Inc.) were dissolved in $10 \mathrm{mM}$ sodium phosphate buffer $\mathrm{pH} 7.0$ to a final concentration of 200 or $450 \mu M$. They were then loaded into a syringe for titration into a sample cell containing OsACBP1 $(30 \mu M)$ or OsACBP2 $(17$ or $56 \mu M)$ through 20 successive $1.8 \mu$ injections over $4 \mathrm{~s}$ at $150 \mathrm{~s}$ intervals with a stirring speed of $1000 \mathrm{rev} \mathrm{min}^{-1}$. For data processing, nonspecific heat effects after saturation were estimated and corrected, the baseline was adjusted and the data were fitted to single-site binding models using Origin v.7.0 following the methods in the software manual.

\section{Results}

3.1. Description and basic comparison of the OsACBP1 and OsACBP2 structures

The apo human liver ACBP model was used to solve the OsACBP1 structure since it has the highest sequence identity (52\%) among proteins with known crystal structures (Fig. 1). The crystal structures of OsACBP1 and OsACBP2 contain one and four protein molecules in the asymmetric unit, respectively (Table 1). The four OsACBP2 molecules present in the asymmetric unit offer a snapshot into the dynamics of the OsACBP2 protein. In spite of the $79 \%$ sequence identity between OsACBP1 and OsACBP2 (Fig. 1), important differences between their structures which have potential implications for ligand binding are reported.

In general, the structures show high similarity; the rootmean-square displacement (r.m.s.d.) in backbone atoms between OsACBP1 and each of the four OsACBP2 molecule is $\sim 1.4 \AA$. Within the four OsACBP2 molecules, molecules $A$ and $B$ demonstrate a more similar crystal-packing environment, as do molecules $C$ and $D$, with a backbone r.m.s.d. of $0.2-0.3 \AA$ within each pair and $\sim 0.7 \AA$ across the two groups (Fig. 2a). Regions that display a greater backbone variability (backbone r.m.s.d. of $>2 \AA$ ) between OsACBP1 and OsAcBP2 include residues 19-20, 44-52, 65-66 and the C-terminus, with a highest r.m.s.d. of $>4.8 \AA$ at residues 51-52 (Fig. $2 b$ ).

Both the OsACBP1 and OsACBP2 structures consist of four helices (Fig. 3), denoted $\mathrm{H} 1-\mathrm{H} 4$ hereafter, adopting the same ACBP scaffold as seen in other structures of ACBPs determined to date. $\mathrm{H} 1$ extends from the first native residue (denoted residue 1) to residue 14 in OsACBP1 and from the first observable residue in the electron density (encompassing residues from part of the $\mathrm{His}_{6}$ tag in molecules $C$ and $D$ of OsACBP2) to residues 13 (molecule $D$ ), 14 (molecule $C$ ) or 15 (molecules $A$ and $B$ ) in OsACBP2. H2 consists of residues 2338 in OsACBP1 and 23-37 in OsACBP2. H4 forms the longest helix, spanning residues 70-89 in OsACBP1 and residues 6886 or $68-87$ in the four OsACBP2 molecules. H3 is shorter

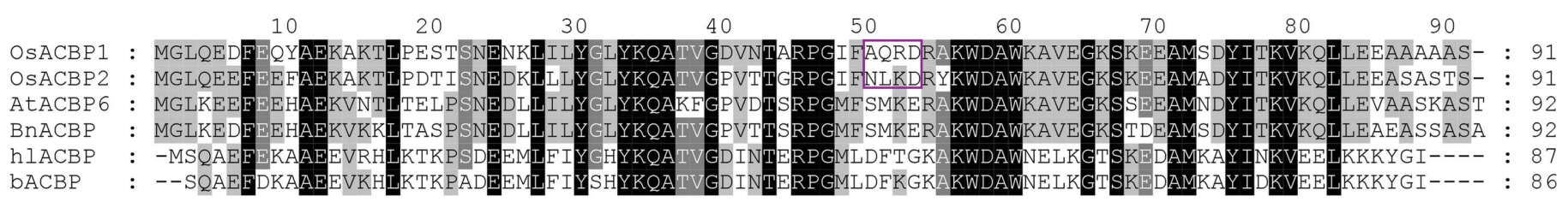

Figure 1

Sequence alignment of OsACBP1 and OsACBP2 from O. sativa L. ssp. japonica, AtACBP6 from A. thaliana, B. napus $10 \mathrm{kDa}$ ACBP (BnACBP), human liver ACBP (hlACBP) and bovine ACBP (bACBP). The numbering at the top corresponds to OsACBP1 and OsACBP2. The alignment was performed using Clustal Omega (Sievers et al., 2011) and formatted in GeneDoc (v.2.6; Nicholas et al., 1997). Amino acids that are identical in all proteins are shaded black, those that are identical in five proteins dark grey, and those that are identical in four proteins light grey. Residues 50-53 in OsACBP1 and OsACBP2 are boxed with a purple line to highlight their differences. 
in OsACBP1 (residues 54-61) than in OsACBP2 (residues 51-62) as OsACBP1 lacks a complete helical turn at the $\mathrm{N}$-terminus of this helix. $\mathrm{H} 1$ and $\mathrm{H} 2$ are antiparallel to each other, as are $\mathrm{H} 3$ and $\mathrm{H} 4$, but $\mathrm{H} 2$ and $\mathrm{H} 3$ are parallel. The helices are joined by loops designated the $\mathrm{H} 1-\mathrm{H} 2$ loop, $\mathrm{H} 2-$ $\mathrm{H} 3$ loop and $\mathrm{H} 3-\mathrm{H} 4$ loop.

Differences between OsACBP1 and OsACBP2 are immediately manifested when the four OsACBP2 molecules are separately superimposed onto OsACBP1 (Fig. 4a). Within residues 50-53, where the amino-acid sequence is AQRD in OsACBP1 and NLKD in OsACBP2 (Fig. 1), residues 51-53 are no longer helical in OsACBP1 and the backbone of this region has shifted 5-6 $\AA$ away from the corresponding region in OsACBP2 toward the periphery (Fig. 4b). The loop before $\mathrm{H} 3$ (residues 44-49) and that between $\mathrm{H} 1$ and $\mathrm{H} 2$ are also highly variable, and the equivalent regions of bovine ACBP have been shown to be less well defined in NMR studies (Kragelund et al., 1993). In the crystal structures, the flexibility

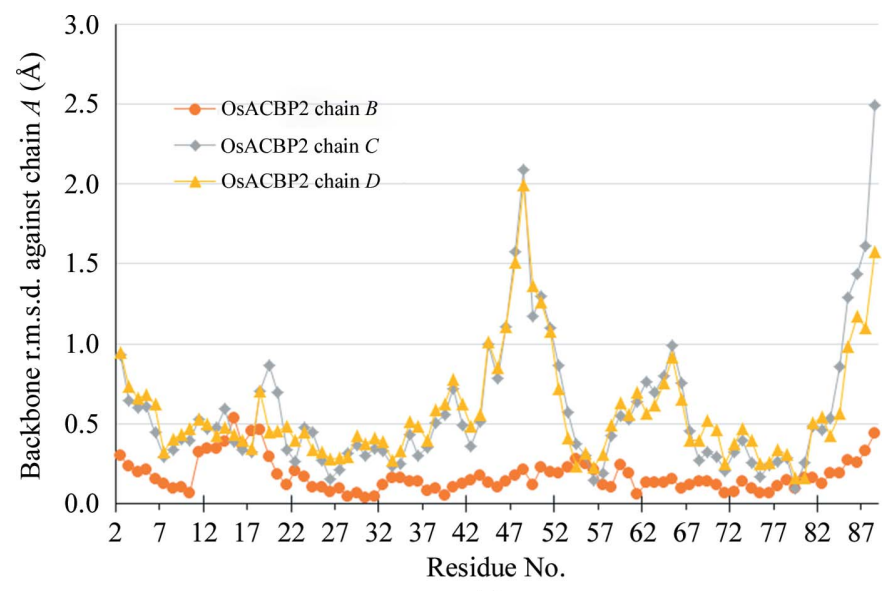

(a)

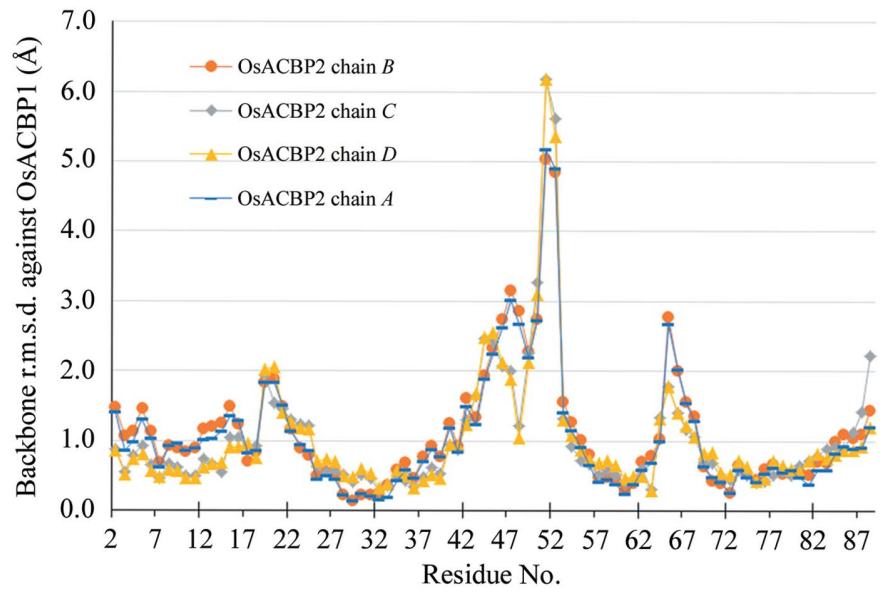

(b)

Figure 2

Root-mean-square displacements (r.m.s.d.s) in backbone atoms per residue based on pairwise calculations of (a) OsACBP2 chains $B, C$ and $D$ against chain $A$ and $(b)$ OsACBP2 chains $A, B, C$ and $D$ against OsACBP1. OsACBP2 chains $A, B, C$ and $D$ are plotted as blue dashes, orange circles, grey diamonds and amber triangles, respectively, in the graphs. The values were calculated using MultiSeq (Roberts et al., 2006) in VMD (Humphrey et al., 1996). of the start of $\mathrm{H} 3$ and the immediately preceding region is reflected by the variability of the backbone among the four molecules of OsACBP2 (Fig. 4b), with markedly higher backbone $B$ factors of residues $47-53$ in OsACBP1 and molecules $A$ and $B$ in OsACBP2 (20-60\% higher than the average) as well as more discontinuous electron density of the side chains (illustrated by OsACBP1 in Fig. 4c).

\subsection{Surface features of OsACBP1 and OsACBP2}

The effects of the differences at the start of $\mathrm{H} 3$ between OsACBP1 and OsACBP2 became more evident when the molecular surfaces of both proteins were displayed and the electrostatic potentials were mapped onto these surfaces. In OsACBP2 there is a deep (depth 8-9 $\AA$ ) and narrow (width 9$10 \AA$ ) groove, which is largely hydrophobic in nature, through the 'front' and 'back' sides in the centre of the 'top' portion of the protein between the N-termini of $\mathrm{H} 3$ and $\mathrm{H} 2$ (Fig. $5 a$ ). It resembles the surface representation of human liver ACBP (Taskinen et al., 2007), which uses a similar groove for ligand binding (Fig. $5 b$ ). In contrast, this top central groove in OsACBP1 has become wider (width 17-18 ), shallower (depth 4-5 $\AA$ ) and more solvent-exposed towards the 'back' side. A space has opened up, created by the new conformation of residues 50-53, which have moved away from the central groove (Fig. 5c). In this respect, it resembles bovine ACBP in the apo form, the 'top' part of which also features a shallower groove (Fig. 5d). Furthermore, the movement of these residues has exposed the negatively charged residues Asp53 and Asp58 to the lining of the groove in OsACBP1, while these residues are shielded from above by neutral residues in OsACBP2. This may confer different ligand-binding properties (affinity and mode) on OsACBP1. Bovine ACBP and human liver ACBP have been chosen for comparison because they are the only ACBPs for which both apo and ligand-bound structures have been determined, while their ligand-binding modes have been shown to be distinct.

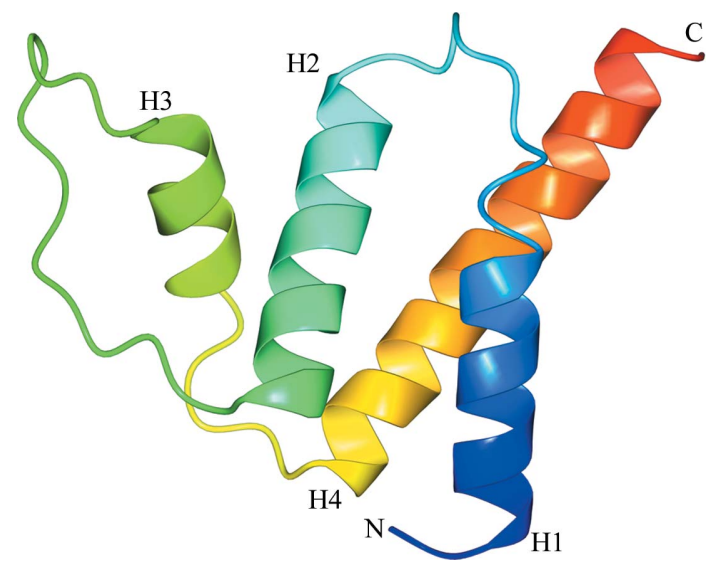

Figure 3

The structure of OsACBP1 in ribbon representation, coloured using a gradual transition from blue at the $\mathrm{N}$-terminus (labelled $\mathrm{N}$ ) to red at the C-terminus (labelled $C$ ). The four helices $(\mathrm{H} 1-\mathrm{H} 4)$ are labelled at their N-termini. 
3.3. Prediction of the ligand-binding modes of OsACBP1 and OsACBP2

To gain a better insight into their function, potential ligandbinding cavities and residues in OsACBP1 and OsACBP2 were predicted using the MetaPocket 2.0 web server (Zhang et $a l ., 2011)$. To confirm its utility, it was first tested on the apo structures of bovine ACBP (Kragelund et al., 1993; PDB entry 2abd; model 1 of the NMR ensemble was used) and the human liver ACBP monomer (Taskinen et al., 2007; PDB entry 2fj9) for comparison with their respective ligand-bound structures. The ligand-binding cavities were found to coincide with a large part of the 16:0-CoA ligand in bovine ACBP (PDB entry 1aca;

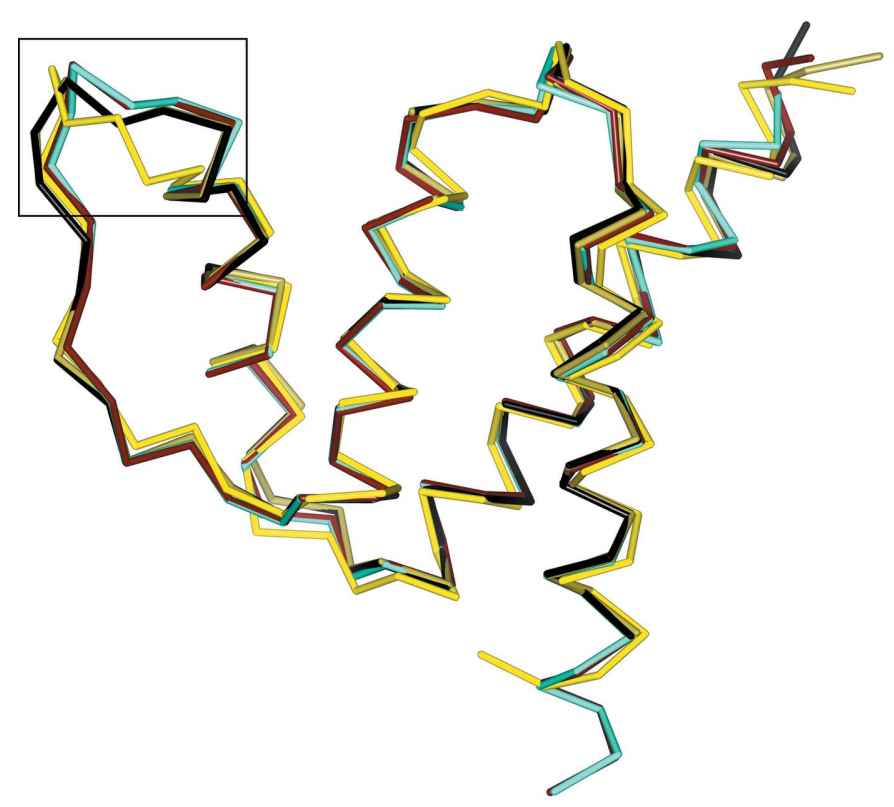

(a)

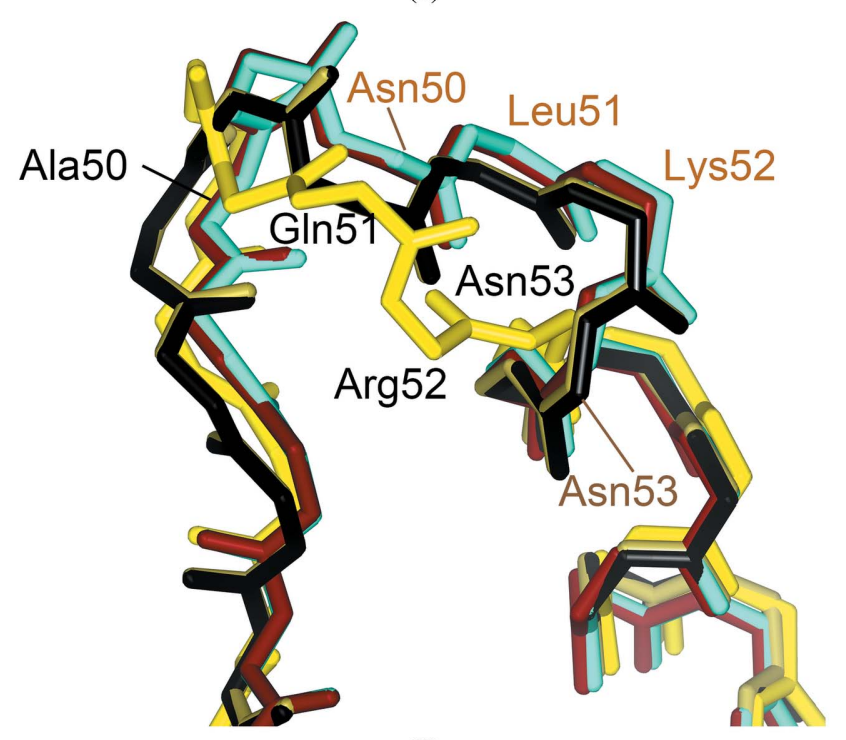

(b)
Kragelund et al., 1993) and the only complete ligand molecule, myristoyl-CoA [14:0-CoA; called C1 in Taskinen et al. (2007) and assigned as residue B1088 in PDB entry 2cb8] in human liver ACBP (data not shown). The overall structural changes between the apo and ligand-bound forms are minimal in both human liver and bovine ACBP, which should also be applicable to OsACBP1 and OsACBP2 in view of the sequence and structural similarities of these ACBPs (Figs. 1 and 5) and thus provide a basis for the prediction of ligand-binding cavities.

The two top-ranked ligand-binding cavities of OsACBP2, represented by molecule $A$, formed one continuous cavity which coincided with the top central groove and largely matched the binding mode of the C1 14:0-CoA molecule observed in PDB entry $2 \operatorname{cb} 8$ (Fig. $6 a$ ), especially in the acyl moiety. The predicted OsACBP2 ligand-binding residues were found to be clustered from the middle of $\mathrm{H} 1$ to the middle of the H1-H2 loop (residues 7-19), the start to the middle of $\mathrm{H} 2$ (residues 23-33) and the start to the middle of $\mathrm{H3}$ (residues 51-55), in agreement with the actual residues in contact $(<5 \AA)$ with the C1 14:0-CoA molecule in human liver ACBP chain $A$, comprising the middle of $\mathrm{H} 1$, the start to middle of $\mathrm{H} 2$ and the start to middle of $\mathrm{H} 3$, as well as residue 44 in the $\mathrm{H} 2-\mathrm{H} 3$ loop. In contrast, the predicted ligand-binding cavity of OsACBP1 resembles bovine ACBP with regard to the proximity to the $\mathrm{H} 1-\mathrm{H} 2$ loop, as well as OsACBP2 in the front part of the top central groove. The predicted OsACBP1 ligand-binding residues were found in the whole $\mathrm{H} 1-\mathrm{H} 2$ loop (residues 14-22) and the start to the middle of $\mathrm{H} 2$ (residues 23-34), as well as residues 53 and 56 in $\mathrm{H} 3$ and Tyr75 in H4 (Fig. 6b). The actual residues in contact with 16:0-CoA in bovine ACBP include a continuous stretch from the middle of $\mathrm{H} 1$ to the middle of $\mathrm{H} 2$ and the start to the middle of $\mathrm{H} 3$, as

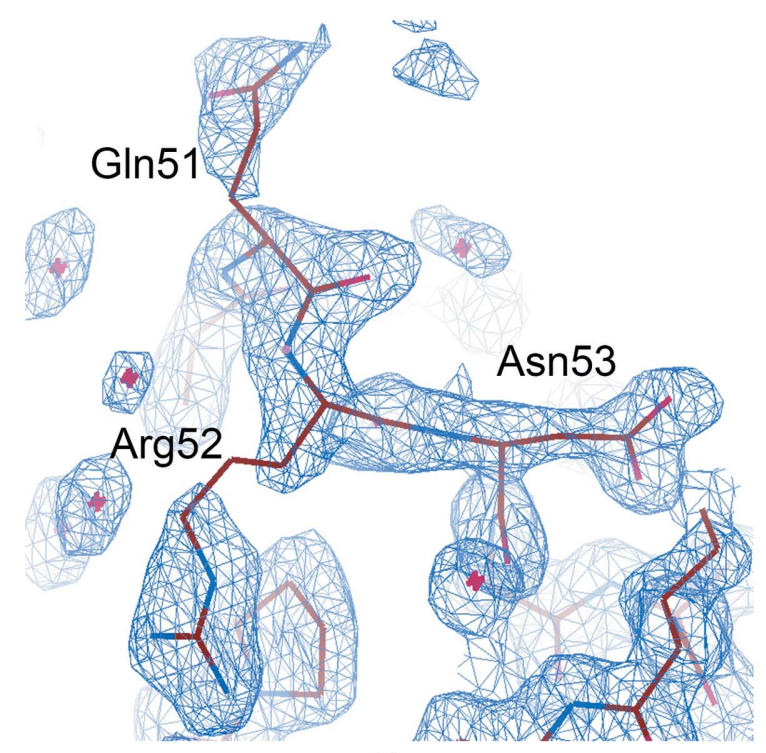

(c)

Figure 4

Variability at the start of $\mathrm{H} 3$ and its preceding loop in OsACBP1 and OsACBP2. (a) Comparison of the OsACBP2 and OsACBP1 structures shown as $\mathrm{C}^{\alpha}$ traces by superimposing each of the four OsACBP2 molecules ( $A$ in pale gold, $B$ in black, $C$ in cyan and $D$ in tan) onto OsACBP1 (in yellow). Residues 50-53 are boxed. (b) Enlargement of the backbone trace in the vicinity of residues 50-53, showing the displacement and loss of helical conformation in OsACBP1. The residues are labelled indicating their $\mathrm{C}^{\alpha}$ atoms: OsACBP1 with black labels and OsACBP2 with brown labels. (c) The electron density $\left(2 F_{\mathrm{o}}-F_{\mathrm{c}}\right.$ contoured at the $1 \sigma$ level $)$ showing discontinuity in the side-chain density of residues 51 and 52 in OsACBP1. 
well as Tyr73 (equivalent to Tyr75 in OsACBP1). The involvement of $\mathrm{H} 3$ is predicted to be less extensive in OsACBP1 than in OsACBP2, while the $\mathrm{H} 1-\mathrm{H} 2$ loop may play a greater role in ligand binding in OsACBP1.

\subsection{Isothermal titration calorimetry (ITC)}

ITC experiments on the binding of $\mathrm{His}_{6}-\mathrm{OsACBP} 1$ and $\mathrm{His}_{6}-\mathrm{OsACBP} 2$ to various acyl-CoA esters were conducted to determine the similarities and differences in their biochemical functions. 16:0-CoA, 18:2-CoA and 18:3-CoA were tested (Fig. 7) as they are some of the most common acyl-CoA esters present intracellularly in plants (Ohlrogge \& Browse, 1995). All injections of acyl-CoA esters into protein solutions were exothermic in nature and the magnitudes of heat release were similar across all experiments. A single-site binding model was chosen using Origin, as it showed a proper fit and is justifiable based on the available ligand-bound ACBP structures. Thermodynamic parameters are listed in Table 2, including the stoichiometry of ligand-protein binding $(n)$, enthalpy change $(\Delta H)$, entropy change $(\Delta S)$ and dissociation constant $\left(K_{\mathrm{d}}\right)$. Both proteins showed moderate to high affinities for the tested acyl-CoA esters, with $K_{\mathrm{d}}$ values ranging from 0.031 to $2.36 \mu \mathrm{M}$ for OsACBP1 and 0.080 to $0.85 \mu M$ for OsACBP2 (Table 2). OsACBP2 displayed a generally higher affinity towards unsaturated acyl-CoA esters than OsACBP1, while the binding affinities and energetics for the saturated 16:0-CoA ester were highly similar for both proteins (Table 2). Both proteins may undergo oligomerization when bound to 16:0-CoA and 18:3$\mathrm{CoA}$ but remain monomeric in complex with $18: 2-\mathrm{CoA}$, as suggested by the binding stoichiometries that were observed.

\section{Discussion}

This paper describes the first structural determination of ACBPs from a plant, representing a new approach to discern their functions in plants. OsACBP1 and OsACBP2 were chosen in this study in order to understand why two highly similar ACBPs are required in rice, an important staple crop. A comparison of the structures, molecular surfaces, predicted ligand-binding modes and actual ligand-binding results from ITC has revealed that OsACBP1 and OsACBP2 are structurally and functionally distinct despite their high sequence identity. The most salient structural difference between OsACBP1 and OsACBP2 lies in the missing helical turn at the N-terminus of $\mathrm{H} 3$ in OsACBP1. Residues 51-53,

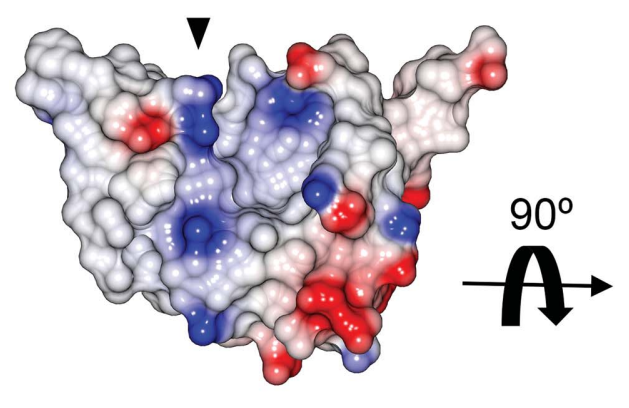

(a)

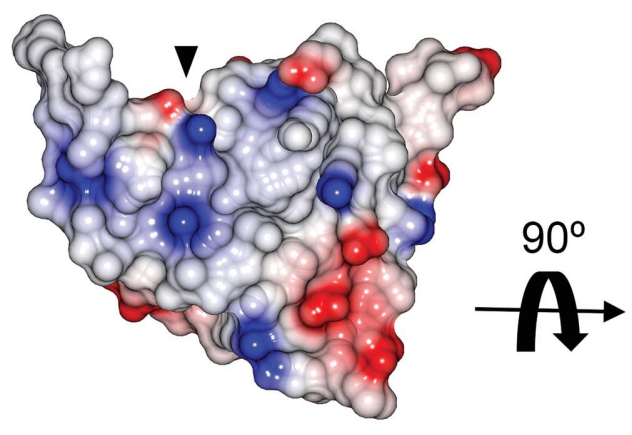

(b)
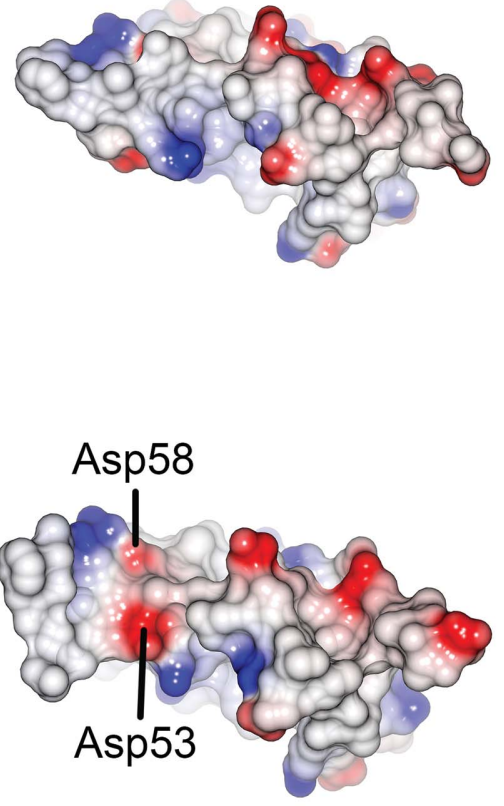

)

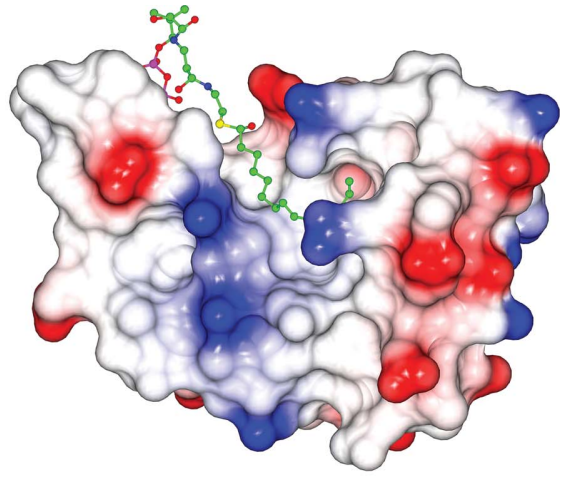

(b)

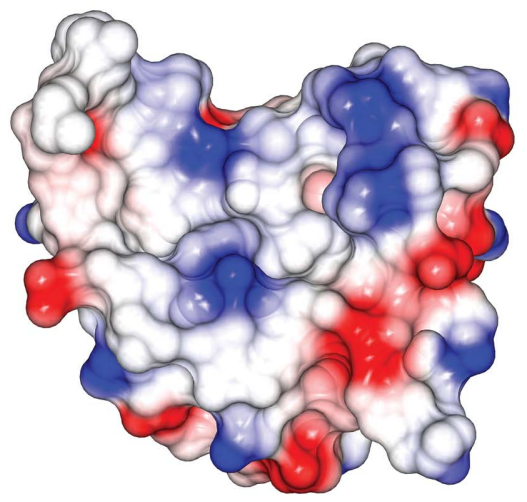

$(d)$

Figure 5

Electrostatic molecular surfaces of OsACBP1, OsACBP2, bovine ACBP and human liver ACBP calculated using default values and as displayed by the molecular-graphics program CCP4mg (McNicholas et al., 2011). Positively charged, neutral and negatively charged regions are coloured blue, white and red, respectively. (a) Molecular surface of OsACBP2 in two views, with the right panel a $90^{\circ}$ rotation from the left panel, showing a 'top-down' view. The top central groove is marked with an arrowhead. (b) Molecular surface of human liver ACBP with C1 (myristoyl-CoA) depicted as a ball-and-stick model, illustrating how this groove is utilized for ligand binding. (c) Molecular surface of OsACBP1 in two views, with the right panel a $90^{\circ}$ rotation from the left panel, showing a 'top-down' view. Residues Asp53 and Asp58 are labelled. The top central groove is marked with an arrowhead. (d) Molecular surface of bovine ACBP in the apo form. There is a similarity between bovine ACBP and OsACBP1 in that the top central groove is shallow. 
Table 2

Thermodynamic parameters for acyl-CoA binding to $\mathrm{His}_{6}-\mathrm{OsACBP} 1$ and $\mathrm{His}_{6}$-OsACBP2.

ITC experiments were carried out at $303 \mathrm{~K}$ in $10 \mathrm{~m} M$ sodium phosphate buffer $\mathrm{pH}$ 7.0. The values and errors are means of three experiments. $n$, stoichiometry; $\Delta H$, enthalpy change; $\Delta S$, entropy change; $\Delta G$, Gibbs free energy; $K_{\mathrm{d}}$, dissociation constant.

\begin{tabular}{clllll}
\hline Acyl-CoA & $n$ & $\begin{array}{l}\Delta H \\
\left(\mathrm{kcal} \mathrm{mol}^{-1}\right)\end{array}$ & $\begin{array}{l}\Delta S \\
\left(\mathrm{cal} \mathrm{mol}^{1} \mathrm{~K}^{1}\right)\end{array}$ & $\begin{array}{l}\Delta G \\
\left(\mathrm{kcal} \mathrm{mol}^{-1}\right)\end{array}$ & $\begin{array}{l}K_{\mathrm{d}} \\
(\mu M)\end{array}$ \\
\hline OsACBP1 & & & & & \\
$16: 0$ & $0.51 \pm 0.00$ & $-12.5 \pm 0.1$ & $-6.96 \pm 0.67$ & $-10.5 \pm 0.1$ & $0.028 \pm 0.006$ \\
$18: 2$ & $1.27 \pm 0.02$ & $-6.31 \pm 0.14$ & $5.02 \pm 0.48$ & $-7.84 \pm 0.05$ & $2.36 \pm 0.19$ \\
$18: 3$ & $0.44 \pm 0.02$ & $-6.58 \pm 0.35$ & $4.66 \pm 1.18$ & $-7.99 \pm 0.05$ & $1.83 \pm 0.20$ \\
OsACBP2 & & & & & \\
$16: 0$ & $0.33 \pm 0.01$ & $-10.9 \pm 0.3$ & $-4.18 \pm 1.19$ & $-9.66 \pm 0.16$ & $0.11 \pm 0.04$ \\
$18: 2$ & $0.92 \pm 0.02$ & $-8.46 \pm 0.17$ & $-0.03 \pm 0.59$ & $-8.46 \pm 0.05$ & $0.85 \pm 0.07$ \\
$18: 3$ & $0.51 \pm 0.01$ & $-10.2 \pm 0.2$ & $-1.09 \pm 0.87$ & $-9.90 \pm 0.12$ & $0.080 \pm 0.019$ \\
\hline
\end{tabular}

three possibilities: (i) these residues undergo movement that will bring them into contact with the ligand upon binding, (ii) the altered topology and surface properties of the ligand-binding site can accommodate ligands in such a way that these residues can interact with ligands without needing significant movement or (iii) there is a novel ligand-binding mode that does not require these residues. The first possibility is based on observations in bovine ACBP, in which residues $46-50$, immediately before and at the start of $\mathrm{H} 3$, move inward by $3-4 \AA$ from the apo

which form the start of $\mathrm{H} 3$ in OsACBP2, have lost their helical conformation and have become part of the $\mathrm{H} 2-\mathrm{H} 3$ loop in OsACBP1 (Fig. 4). This is accompanied by a movement of these residues away from the predicted ligand-binding site and is attributable to differences in the amino-acid sequence at residues 50-52. The surface properties (Fig. 5) and predicted ligand-binding cavities (Fig. 6) also differ between the two ACBPs. OsACBP2 highly resembles human liver ACBP, in which the ACBP dimerizes and the ligand spans both ACBP molecules, whereas the ligand-binding site of OsACBP1 is predicted to involve similar residues to bovine ACBP but displays some similar features to those of OsACBP2.

The bovine and human liver ACBP structures have revealed that the start to the middle of $\mathrm{H} 3$ is an integral part of the ligand-binding site. In the current state, the residues that are part of the missing first turn of $\mathrm{H} 3$ in OsACBP1 do not appear to be able to participate in ligand binding. This may arise from form to bring residues 49 and 50 closer to the bound 16:0-CoA (Kragelund et al., 1993). It may also be applicable to OsACBP1 in view of the higher flexibility of the corresponding region in OsACBP1 (residues 49-53, where the backbone $B$ factors are $30-50 \%$ higher than the average backbone $B$ factor), and the similarity between the predicted ligand-binding mode in OsACBP1 and the actual mode in bovine ACBP.

ITC experiments uncovered further differences between OsACBP1 and OsACBP2 in their acyl-CoA-binding properties despite their high sequence identity. OsACBP2 displayed submicromolar $K_{\mathrm{d}}$ values for the acyl-CoA esters tested, while in OsACBP1 the $K_{\mathrm{d}}$ values ranged from $31 \mathrm{n} M$ for 16:0-CoA to around $2 \mu M$ for the unsaturated acyl-CoAs (Table 2). Such a distinctive binding preference stands in contrast to AtACBP6, the other class I member, which exhibits similar binding affinities $\left(K_{\mathrm{d}}=36-84 \mathrm{n} M\right)$ for a range of saturated and

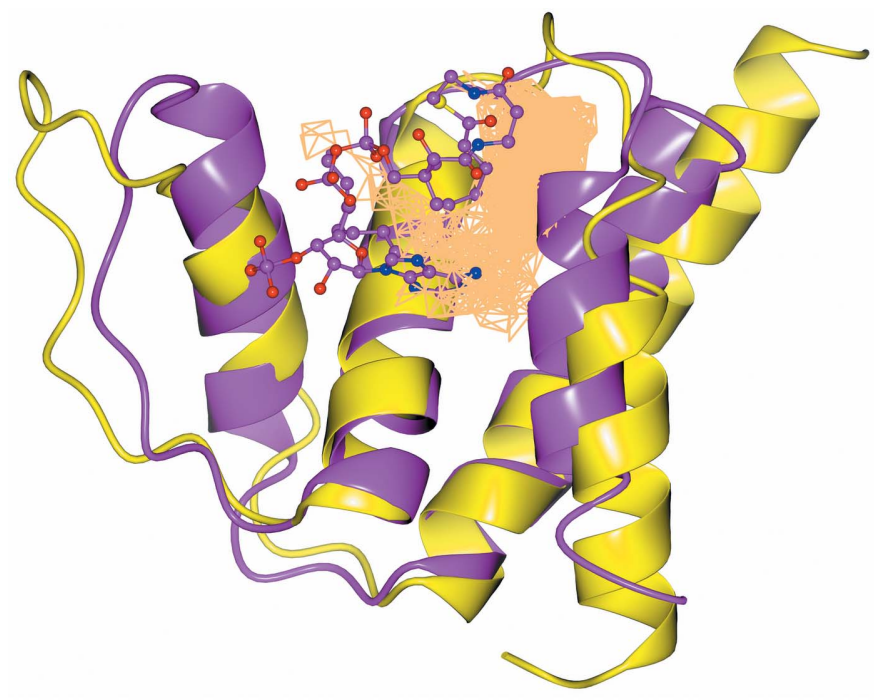

(a)

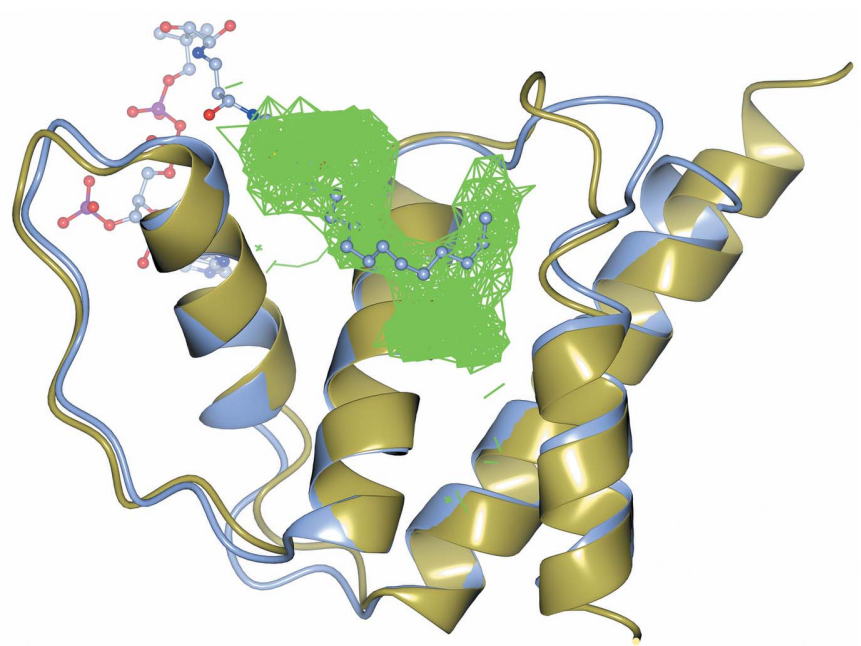

(b)

Figure 6

Predicted ligand-binding sites in OsACBP1 and OsACBP2 and a comparison with the actual sites in bovine and human liver ACBP, respectively. (a) OsACBP1 and bovine ACBP are displayed as yellow and magenta ribbons, respectively; the ligand (palmitoyl-CoA) of bovine ACBP is shown as a balland-stick model. The predicted binding cavity of OsACBP1 is shown as a pale orange patch. Its proximity to the H1-H2 loop is in line with the involvement of this loop in ligand binding to bovine ACBP. (b) OsACBP2 molecule $A$ and human liver ACBP are displayed as pale-gold and ice-blue ribbons, respectively; the ligand (C1 myristoyl-CoA) of human liver ACBP is shown as a ball-and-stick model. The predicted binding cavity of OsACBP2 is shown as a green patch and overlaps with a large part of the acyl chain of the myristoyl-CoA. 
unsaturated acyl-CoAs (Hsiao et al., 2014). Furthermore, some of the binding interactions gave a negative entropic change, which may arise when, for example, increased rigidity in the protein and ligand upon ligand binding or protein dimerization outweighs the entropy contribution of other effects.

The structures of OsACBP1 and OsACBP2 add insight to how the structure and function of members of the ACBP family may diversify despite their overall high sequence similarity and the general conservation of the four-helix core structure. OsACBP1 and OsACBP2 are localized to the cytosol as GFP-tagged fusions (Meng et al., 2014). One major function of plant cytosolic ACBPs is modulation of the intracellular distribution of lipids through transporting acylCoA esters and protecting them from hydrolysis (Engeseth $e t$ al., 1996), as suggested by experiments on various class I ACBPs. In Arabidopsis, the AtACBP6 mutant displays increases of 18:1-CoA and 18:2-CoA in five-day-old seedlings and a greater accumulation of 18:2-CoA in cotyledon-staged embryos compared with the wild type (Hsiao et al., 2014). B. napus $10 \mathrm{kDa} \mathrm{ACBP}$ has been shown to facilitate acyl exchange between acyl-CoA and phosphatidylcholine in vitro (Yurchenko et al., 2009), and when heterologously expressed

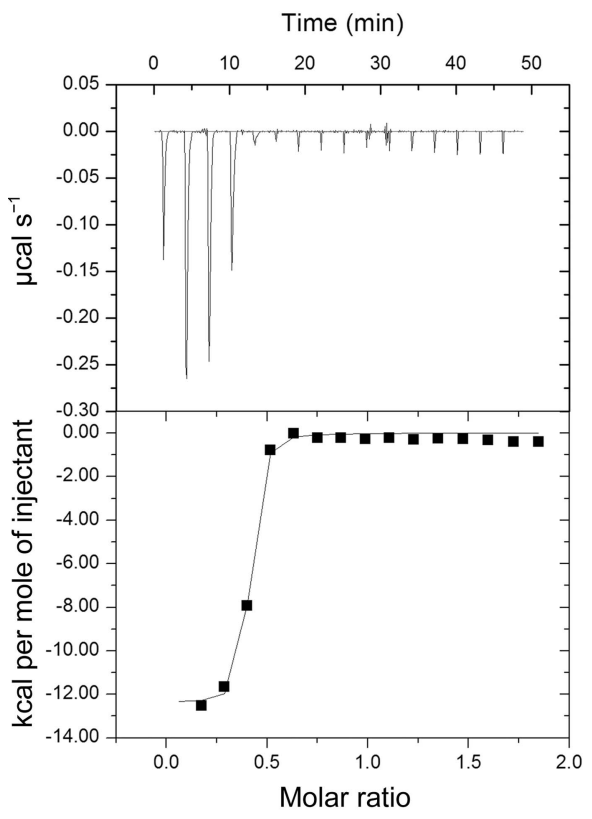

(a)

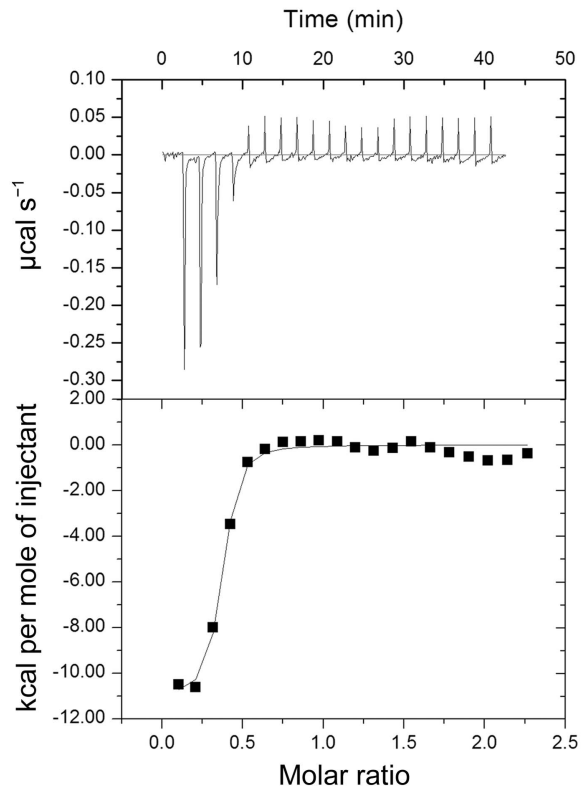

(d)

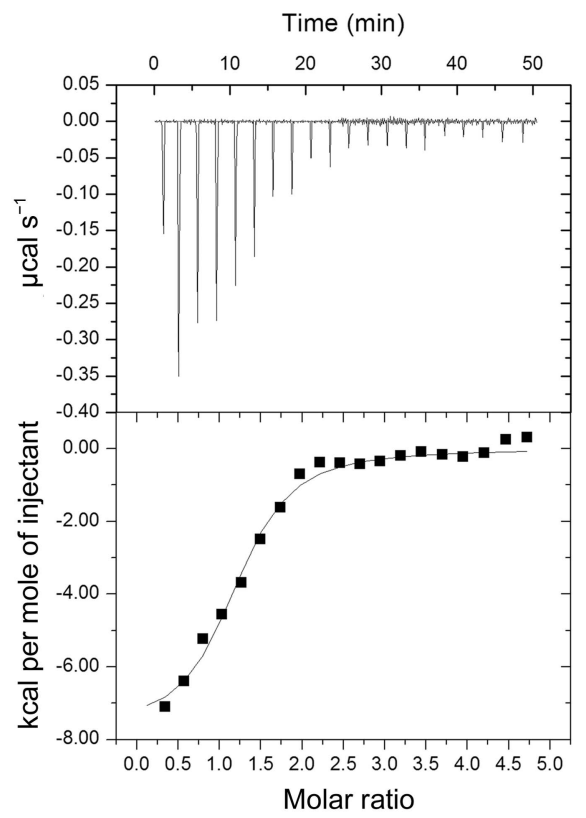

(b)

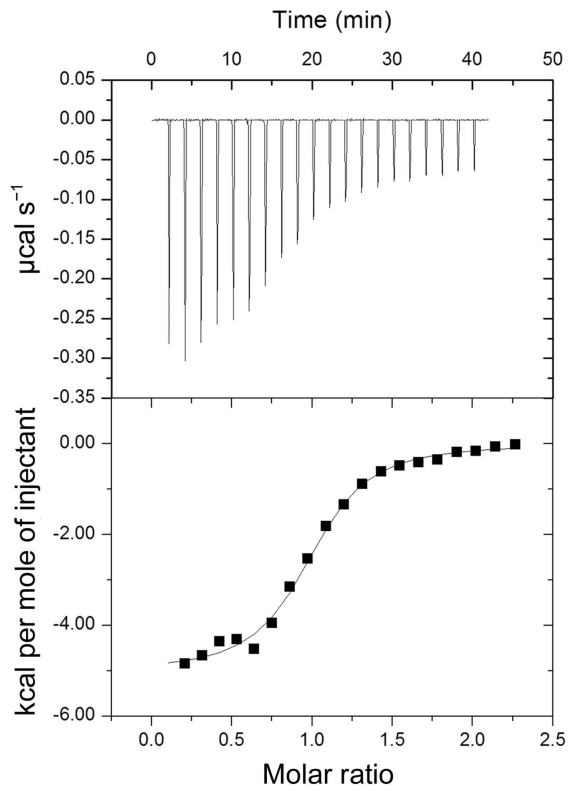

(e)

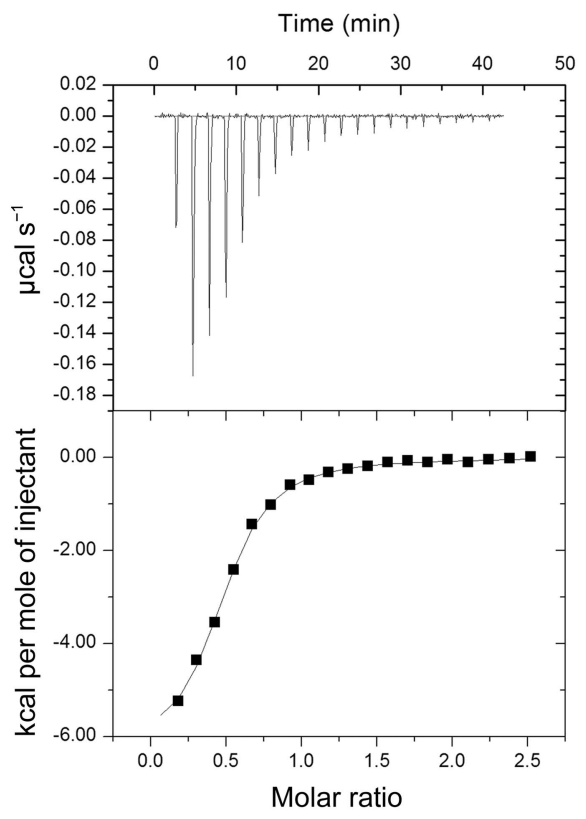

(c)

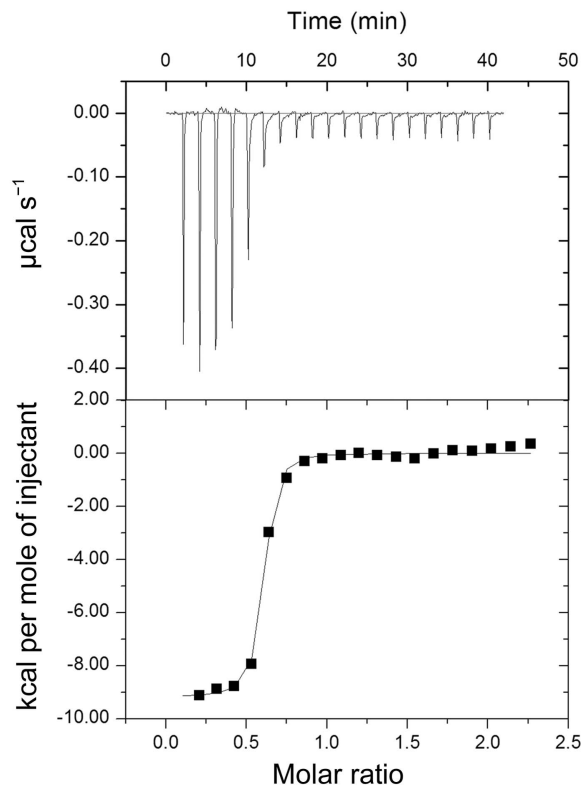

(f)

Figure 7

Binding isotherms from isothermal titration calorimetry of OsACBP1 and OsACBP2 with acyl-CoA esters. (a) Isotherm of $30 \mu M$ OsACBP1 with $450 \mu M$ 16:0-CoA (palmitoyl-CoA). (b) Isotherm of $30 \mu M$ OsACBP1 with $450 \mu M$ 18:2-CoA (linoleoyl-CoA). (c) Isotherm of $30 \mu M$ OsACBP1 with $450 \mu M$ 18:3-CoA (linolenoyl-CoA). (d) Isotherm of $17 \mu M$ OsACBP2 with $200 \mu M$ 16:0-CoA. (e) Isotherm of $17 \mu M$ OsACBP2 with $200 \mu M 18: 2-C o A$. (f) Isotherm of $17 \mu M$ OsACBP2 with $200 \mu M$ 18:3-CoA. 
in Arabidopsis it alters the composition of and equilibrium between the fatty-acid and acyl-CoA pools in developing and mature seeds (Yurchenko et al., 2014). The high sequence conservation between OsACBP1, OsACBP2, AtACBP6 and Brassica ACBP (Fig. 1) would suggest that the two OsACBPs are likely to have a similar general role in modulating lipid metabolism. However, their precise roles may differ as their expression profiles diverge in seed development (Meng et al., 2011). Whereas AtACBP6 is cold-inducible (Chen et al., 2008), OsACBP1, OsACBP2 and OsACBP3 mRNA levels are suppressed within $12 \mathrm{~h}$ of cold treatment before recovery to near-normal levels at $24 \mathrm{~h}$ (Meng et al., 2011). Taken together with the results of varying lipid-binding profiles amongst OsACBP1, OsACBP2 and AtACBP6 from ITC experiments (Hsiao et al., 2014), there appears to be a clear functional diversity of class I ACBPs during seed development and in response to cold stress (Meng et al., 2011).

The predicted ligand-binding cavity in OsACBP2 is likely to be able to accommodate the acyl moiety of bound acyl-CoAs similarly to the conformation adopted by human liver ACBP, given the similarity between the predicted cavity in OsACBP2 and the observed ligand conformation in human liver ACBP. Following the human liver ACBP model in binding myristoylCoA (Taskinen et al., 2007) and results from our ITC experiments, OsACBP1 and OsACBP2 may possibly dimerize upon binding to 16:0-CoA and 18:3-CoA. Further biochemical characterization and structural studies of the proteins in the ligand-bound form would help to confirm this hypothesis.

\section{Acknowledgements}

The authors wish to thank J. W. T. Tam (School of Biological Sciences, University of Hong Kong), S. M. Chan and Dr C. M. C. Lam (School of Biomedical Sciences, University of Hong Kong) for technical support, as well as Dr M. C. Lawrence (Structural Biology Division, Walter and Eliza Hall Institute of Medical Research, Australia) for advice on crystallography. This work was supported by the Wilson and Amelia Wong Endowment Fund, the Research Grants Council of the Hong Kong Special Administrative Region, People's Republic of China (HKU17105615M and Hong Kong PhD Fellowship to WHYC) and the University of Hong Kong (postgraduate studentship to ZHG, postdoctoral fellowship to GKWK and Small Project Funding CRCG 201309176177 to GKWK and MLC).

\section{References}

Aalten, D. M. F. van, Milne, K. G., Zou, J.-Y., Kleywegt, G. J., Bergfors, T., Ferguson, M. A. J., Knudsen, J. \& Jones, T. A. (2001). J. Mol. Biol. 309, 181-192.

Adams, P. D., Grosse-Kunstleve, R. W., Hung, L.-W., Ioerger, T. R., McCoy, A. J., Moriarty, N. W., Read, R. J., Sacchettini, J. C., Sauter, N. K. \& Terwilliger, T. C. (2002). Acta Cryst. D58, 1948-1954.

Andersen, K. V. \& Poulsen, F. (1993). J. Biomol. NMR, 3, 271-284.

Burton, M., Rose, T. M., Faergeman, N. J. \& Knudsen, J. (2005). Biochem. J. 392, 299-307.

Chen, Q.-F., Xiao, S. \& Chye, M.-L. (2008). Plant Physiol. 148, 304-315.
Chen, Q.-F., Xiao, S., Qi, W., Mishra, G., Ma, J., Wang, M. \& Chye, M.-L. (2010). New Phytol. 186, 843-855.

Chye, M.-L. (1998). Plant Mol. Biol. 38, 827-838.

Chye, M.-L., Huang, B.-Q. \& Zee, S. Y. (1999). Plant J. 18, 205214.

Chye, M.-L., Li, H.-Y. \& Yung, M.-H. (2000). Plant Mol. Biol. 44, 711-721.

Costabel, M. D., Ermácora, M. R., Santomé, J. A., Alzari, P. M. \& Guérin, D. M. A. (2006). Acta Cryst. F62, 958-961.

Du, Z.-Y., Chen, M.-X., Chen, Q.-F., Xiao, S. \& Chye, M.-L. (2013a). Plant Cell Environ. 36, 300-314.

Du, Z.-Y., Chen, M.-X., Chen, Q.-F., Xiao, S. \& Chye, M.-L. (2013b). Plant J. 74, 294-309.

Du, Z.-Y., Xiao, S., Chen, Q.-F. \& Chye, M.-L. (2010). Plant Physiol. 152, 1585-1597.

Emsley, P., Lohkamp, B., Scott, W. G. \& Cowtan, K. (2010). Acta Cryst. D66, 486-501.

Engeseth, N. J., Pacovsky, R. S., Newman, T. \& Ohlrogge, J. B. (1996). Arch. Biochem. Biophys. 331, 55-62.

Gao, W., Li, H.-Y., Xiao, S. \& Chye, M.-L. (2010). Plant J. 62, 989-1003.

Gao, W., Xiao, S., Li, H.-Y., Tsao, S.-W. \& Chye, M.-L. (2009). New Phytol. 181, 89-102.

Hsiao, A.-S., Haslam, R. P., Michaelson, L. V., Liao, P., Chen, Q.-F., Sooriyaarachchi, S., Mowbray, S. L., Napier, J. A., Tanner, J. A. \& Chye, M.-L. (2014). Biosci. Rep. 34, 865-877.

Humphrey, W., Dalke, A. \& Schulten, K. (1996). J. Mol. Graph. 14, 33-38.

Kabsch, W. (1976). Acta Cryst. A32, 922-923.

Kabsch, W. (2010). Acta Cryst. D66, 133-144.

Kabsch, W. \& Sander, C. (1983). Biopolymers, 22, 2577-2637.

Kragelund, B. B., Andersen, K. V., Madsen, J. C., Knudsen, J. \& Poulsen, F. M. (1993). J. Mol. Biol. 230, 1260-1277.

Leung, K.-C., Li, H.-Y., Mishra, G. \& Chye, M.-L. (2004). Plant Mol. Biol. 55, 297-309.

Leung, K.-C., Li, H.-Y., Xiao, S., Tse, M.-H. \& Chye, M.-L. (2006). Planta, 223, 871-881.

Li, H.-Y. \& Chye, M.-L. (2003). Advanced Research on Plant Lipids: Proceedings of the 15th International Symposium on Plant Lipids, edited by N. Murata, M. Yamada, I. Nishida, H. Okuyama, J. Sekiya \& W. Hajime, pp. 271-274. Dordrecht: Springer. https://doi.org/ 10.1007/978-94-017-0159-4_63.

Liao, P., Chen, Q.-F. \& Chye, M.-L. (2014). Plant Cell Physiol. 55, 1055-1071.

McCoy, A. J. (2007). Acta Cryst. D63, 32-41.

McNicholas, S., Potterton, E., Wilson, K. S. \& Noble, M. E. M. (2011). Acta Cryst. D67, 386-394.

Meng, W., Hsiao, A.-S., Gao, C., Jiang, L. \& Chye, M.-L. (2014). New Phytol. 203, 469-482.

Meng, W., Su, Y. C. F., Saunders, R. M. K. \& Chye, M.-L. (2011). New Phytol. 189, 1170-1184.

Monzani, P. S., Pereira, H. M., Melo, F. A., Meirelles, F. V., Oliva, G. \& Cascardo, J. C. (2010). Biochim. Biophys. Acta, 1804, 115-123.

Nicholas, K. B., Nicholas, H. B. \& Deerfield, D. W. (1997). EMBnet.news, 4(2), 1-4.

Ohlrogge, J. \& Browse, J. (1995). Plant Cell, 7, 957-970.

Rasmussen, J. T., Faergeman, N. J., Kristiansen, K. \& Knudsen, J. (1994). Biochem. J. 299, 165-170.

Roberts, E., Eargle, J., Wright, D. \& Luthey-Schulten, Z. (2006). BMC Bioinformatics, 7, 1-11.

Sievers, F., Wilm, A., Dineen, D., Gibson, T. J., Karplus, K., Li, W., Lopez, R., McWilliam, H., Remmert, M., Söding, J., Thompson, J. D. \& Higgins, D. G. (2011). Mol. Syst. Biol. 7, 539.

Strong, M., Sawaya, M. R., Wang, S., Phillips, M., Cascio, D. \& Eisenberg, D. (2006). Proc. Natl Acad. Sci. USA, 103, 80608065.

Taskinen, J. P., van Aalten, D. M., Knudsen, J. \& Wierenga, R. K. (2007). Proteins, 66, 229-238. 
Teilum, K., Thormann, T., Caterer, N. R., Poulsen, H. I., Jensen, P. H., Knudsen, J., Kragelund, B. B. \& Poulsen, F. M. (2005). Proteins, 59, 80-90.

Winn, M. D. et al. (2011). Acta Cryst. D67, 235-242.

Xiao, S., Chen, Q.-F. \& Chye, M.-L. (2009). Plant Physiol. Biochem. 47, 926-933.

Xiao, S. \& Chye, M.-L. (2011). Plant Physiol. 156, 2069-2081.

Xiao, S., Gao, W., Chen, Q.-F., Chan, S.-W., Zheng, S.-X., Ma, J., Wang, M., Welti, R. \& Chye, M.-L. (2010). Plant Cell, 22, 14631482.

Xiao, S., Gao, W., Chen, Q.-F., Ramalingam, S. \& Chye, M.-L. (2008). Plant J. 54, 141-151.
Xiao, S., Li, H.-Y., Zhang, J.-P., Chan, S.-W. \& Chye, M.-L. (2008). Plant Mol. Biol. 68, 571-583.

Xue, Y., Xiao, S., Kim, J., Lung, S.-C., Chen, L., Tanner, J. A., Suh, M. C. \& Chye, M.-L. (2014). J. Exp. Bot. 65, 5473-5483.

Yurchenko, O. P., Nykiforuk, C. L., Moloney, M. M., Ståhl, U., Banaś, A., Stymne, S. \& Weselake, R. J. (2009). Plant. Biotech. J. 7, 602-610.

Yurchenko, O., Singer, S. D., Nykiforuk, C. L., Gidda, S., Mullen, R. T., Moloney, M. M. \& Weselake, R. J. (2014). Plant Physiol. 165, 550-560.

Zhang, Z., Li, Y., Lin, B., Schroeder, M. \& Huang, B. (2011). Bioinformatics, 27, 2083-2088. 